Ann. Sci. forest., 1970, 27 (2), 197-223.

\title{
PREMIÈRE APPRÉCIATION DE LA QUALITÉ DU BOIS EN FORÊT PAR UTILISATION DUUN TORSIOMÈTRE
}

\author{
H. POLGE et R. KELLER
}

Station de Recherches sur la Qualité des Bois, Centre national de Recherches forestières, 54-Nancy

Institut national de la Recherche agronomique

\section{RESUME}

Une nouvelle méthode dévaluation de la qualité du bois sur des arbres vivants est décrite ; elle est basée sur l'utilisation d'un torsiomètre qui sert à mesurer le couple nécessaire pour enfoncer une tarière de Pressler à une profondeur donnée à l'intérieur de l'arbre.

II est rendu compte de plusieurs expériences entreprises pour mettre au point la méthodologie la plus appropriée:

L'expérience \& Sivrite $1 \%$ a montré que la meilleure parmi les quatre modalités de mesure qui ont été essayćes est celle appelée « Stabilisé profond \& (tableau 1). Cette même expérience a permis de vérifier que la saison (arbres en sève ou hors sève) n'exerce aucune influence sur le couple de torsion.

Dans l'expérience * Sivrite 2 \%, l'effet de l'affutage de la tarière a été étudié en comparant entre autres une tarière parfaitement affûtée (tarière 1) et la même après une utilisation intensive et continue $(3.000 \mathrm{~cm}$ de sondages réalisés dans un bois dur) : il existe une différence significative entre les couples de torsion pour le Douglas, mais pas pour le Grandis.

Cependant, contrairement à ce qu’on pourrait supposer, les couples étaient plus faibles avec la tarière usće qu'avec la tarière affútée; l'emploi tout à fait anormal de la tarière 2 semble avoir provoqué un polissage, lui aussi exceptionnel, de l'extérieur du corps de la tarière, d’où une réduction des frottements; ainsi, pour une tarière utilisée de façon normale, l'effet de l'affútage est négligeable.

L'influence de l'opérateur a également été étudiée dans l'expérience « Sivrite 2 s; quand les mesures sont faites après stabilisation, on trouve un effet significatif sur le Grandis, mais pas sur le Douglas; les écarts relevés sont dus apparemment à des différences de vitesse de mise en ceuvre du torsiomètre et peuvent être corrigés si nécessaire.

L'expérience * St Avold $*$ a permis dexpliquer pourquoi les corrélations entre la densité et le couple sont plus étroites que celles observées entre la densité et les diverses caractéristiques mécaniques appréciées sur des éprouvettes normalisées; d'une part, les corrélations avec la densité sont meilleures quand les essais sont faits sur des éprouvettes à l'état frais, d'autre part, la résistance à l'avancement de la tarière présente un caractère complexe (compression perpendiculaire et parallèle au fil du bois), et effectivement la densité est davantage liée à une combinaison de plusieurs caractéristiques mécaniques qưà une seule d'entre elles prise isolément.

Le couple de torsion constitue donc une nouvelle caractéristique, que l'on peut mesurer facilement et rapidement sur un grand nombre d'arbres de façon non destructive: il 
permet en particulier de choisir directement en forêt un individu ayant une densité et des caractéristiques mécaniques voisines de la moyenne d'une parcelle donnée; le torsiomètre pourrait également être utilisé pour lamélioration des arbres forestiers, en rendant possible la sélection in situ d'un grand nombre darbres supérieurs au point de vue de la densité du bois.

\section{1. - INTRODUCTION}

La possibilité d'apprécier la dureté d'un arbre sur pied par la mesure directe du couple de torsion qu'il faut exercer pour y enfoncer une tarière de sondage n'a fait jusqu'ici l'objet que de comptes rendus occasionnels (Polge, 1962, 1963).

C'est là cependant une méthode extrêmement simple, non destructive et dont la mise en cuvre ne requiert aucun travail supplémentaire autre que le remplacement momentané du manche de la tarière par un torsiomètre de modèle courant.

De telles déterminations sont faites maintenant de manière systématique à l'occasion de tous les prélèvements à la tarière auxquels procède la Station de Recherches sur la Qualité des Bois, et les renseignements fournis sont toujours très significatifs, en raison notamment des corrélations étonnamment étroites qui lient le couple de torsion ainsi mesuré à la densité du bois. Le caractère général de ces résultats, le fait que cette première appréciation de la qualité du bois obtenue * in situ * soit le plus souvent confirmée par les études ultérieures en Laboratoire, nous ont amenés à entreprendre une analyse plus approfondie des phénomènes en cause dont il est rendu compte dans le présent travail.

Trois expériences successives seront décrites ici :

- L'expérience \& Sivrite $l$ * qui a été entreprise pour déterminer la meilleure façon de mesurer le couple de torsion et également pour voir si l'époque du sondage exerçait une influence sur la résistance à l'enfoncement de la tarière.

- L'expérience \& Sivrite 2 s dont le but principal était d'étudier l'effet * opérateur * et l'effet * affûtage de la tarière s.

- L'expérience * Saint-Avold $>$ qui a servi à mettre en évidence les liaisons entre le couple de torsion et les diverses autres caractéristiques mécaniques.

\section{2. - EXPERIENCE \& SIVRITE I ;}

\section{1. - Matériel et méthodes}

Dans la première partie de cette expérience, qui s'est déroulée en pleine période de sève (juillet 1969), 16 arbres: 8 Douglas et 8 Grandis de 40 ans ont été sondés à quatre reprises par quatre opérateurs utilisant deux tarières différentes.

Pour que les zones de sondage soient aussi semblables que possible. sans cependant être trop rapprochées afin d'éviter tout risque d'interaction entre trous, les quatre points de mesure ont été choisis de façon à s'inscrire sur chaque arbre suivant une hélice. étant séparés les uns des autres par un décalage angulaire denviron $20^{\circ}$ combiné à une différence de hauteur de l'ordre de $10 \mathrm{~cm}$.

A la suite de certaines erreurs intervenues dans la réalisation du dispositif envisagé, il n’a pas été possible de faire la part des effets * arbre $\$$. * opérateur $*$ et * tarière $»$ dans la variabilité totale, et la question a été reprise à l'occasion de lexpérience suivante \& Sivrite 2 \%. 
Pour le reste, il a été procédé comme suit:

Chaque tarière était munie d'un curseur que l'on plaçait à une distance de la fin des épaulements qui terminent la partie filetée égale à l'épaisseur de l'écorce mesurée au millimètre près à laide d'une jaugc appropriée (voir figure 1); ainsi, lorsque le curseur atteignait le rhytidome externe. On était sûr que la tarière était enfoncée dans le bois jusqu'à la fin des épaulements.

Deux mesures du couple de torsion étaient alors prises, l'une au démarrage de la tarière, la seconde lorsque laiguille du torsiomètre était stabilisée; les mèmes déterminations étaient faites trois tours de tarière plus loin, c'est-à-dire après un enfoncement de $37,5 \mathrm{~mm}$ à l'intérieur du bois. Ces quatre mesures seront appelées respectivement par la suite : couple démarrage superficiel, couple stabilisé superficiel, couple démarrage profond et couple stabilisé profond.

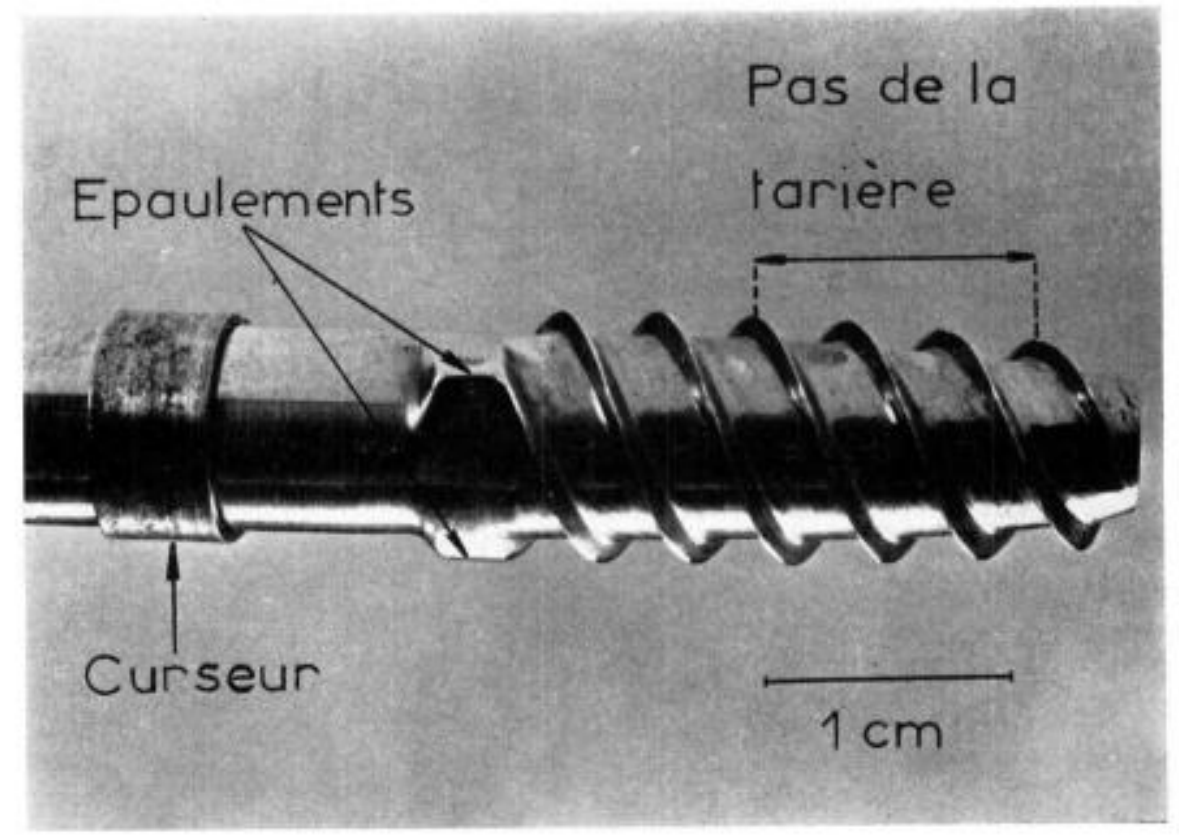

Fia, 1. - Extrémité d'une tarière de $5 \mathrm{~mm}$ munie d'un curseur

F16. 1. - End of an $5 \mathrm{~mm}$ increment borer with its index of bark thickness

Le torsiomètre utilisé était un modèle standard, de capacité $300 \mathrm{~cm} . \mathrm{kg}$, dusage courant en mécanique générale. Grâce à quelques modifications mineures (échelle de graduation rapprochée de l'extrémité de l'aiguille et amincissement de celle-ci pour éviter toute erreur en cas de lecture oblique), la précision des mesures était de $5 \mathrm{~cm} . \mathrm{kg}$ (figure 2).

Dans les carottes de sondage obtenues, ont été découpés deux échantillons de $3 \mathrm{~cm}$ de longueur chacun représentant de façon exacte la zone de bois comprimée par l'ensemble du filetage et des épaulements, d'une part lors des mesures superficielles, d'autre part, lors des mesures profondes. Ils ont été utilisés pour la détermination (par la méthode de saturation intégrale) de la densité du bois en chacun des deux points considérés.

Une nouvelle série de mesures du couple de torsion a été entreprise hors sève (janvier 1970) sur les 8 Grandis de l'expérience pour étudier l'influence éventuelle de l'ćpoque du sondage. Trois seulement des quatre opérateurs ont pu y participer ; ils ont utilisé les deux mêmes tarières qu'au mois de juillet, et les prélèvements ont été faits par chacun d'eux sur les mêmes arbres, aux mêmes niveaux que pour lexpérience en séve, mais avec un léger déplacement angulaire. 


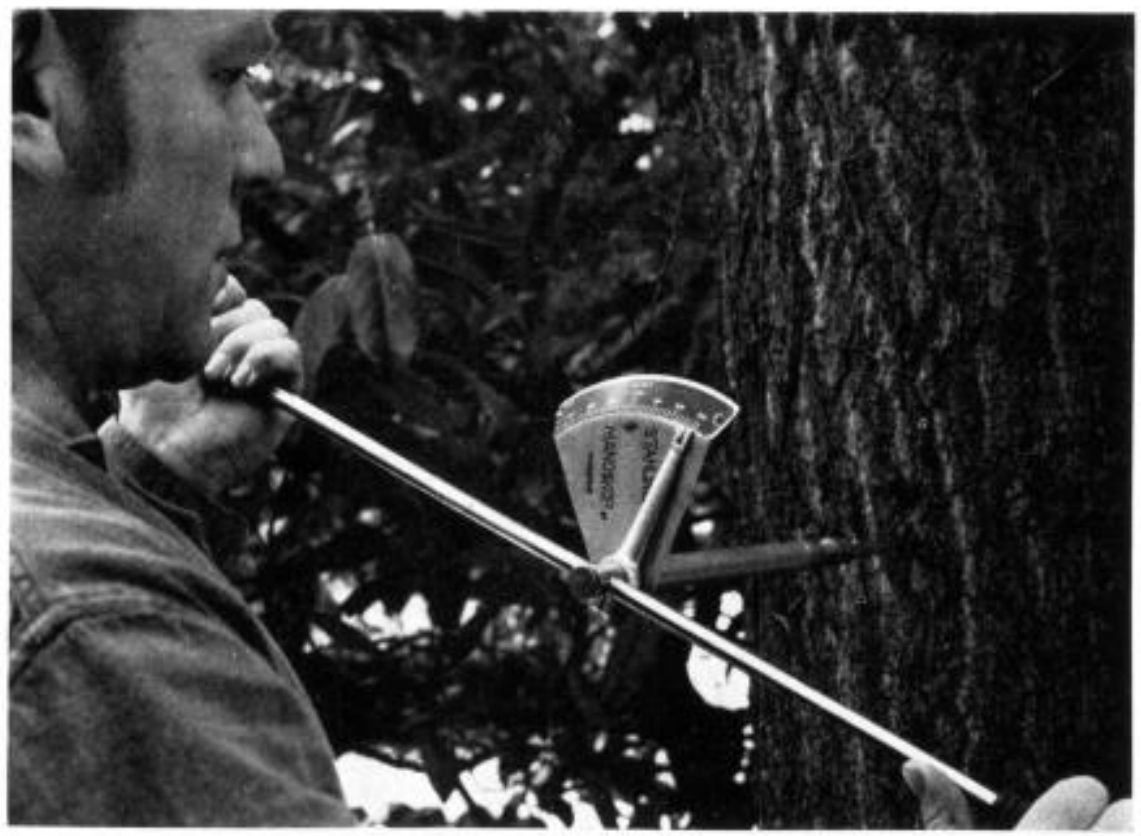

Fici. 2, - Mesure d'un couple de torsion de $285 \mathrm{~cm}, \mathrm{~kg}$ Fic. 2. - Measurememt of a torsion torque of $285 \mathrm{~cm}, \mathrm{~kg}$

TABLEAU ।

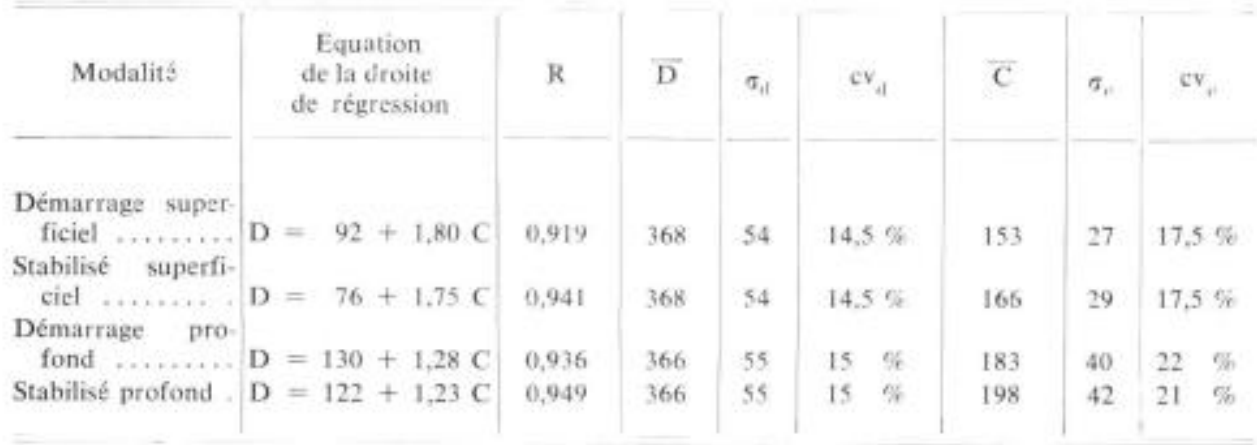

Modalitó : method of measurement.

Dèmarrage superficiel : starting for shallow measurement.

Stabilisé superficiel: shallow measurement after stabilization of the torsiometer.

Démarrage profond : starting for deep measurement.

Stabilisé profond: deep measurement after stabilization.

Equation de la droite de régression: regression line equation. 


\section{2. - Résultats}

2.21. - Choix de la meilleure méthode de mesure.

Le critère retenu pour comparer entre elles les diverses modalités de lecture du torsiomètre est tout naturellement létroitesse de la liaison qui lie le couple de torsion (C) à la densité du bois (D).

Le tableau 1 ci-contre donne, dans chaque cas, l'équation de la droite de régression de $\mathrm{D}$ en fonction de $\mathrm{C}$, le coefficient de corrélation, les valeurs moyennes des deux variables, leur écart-type et leur coefficient de variation.

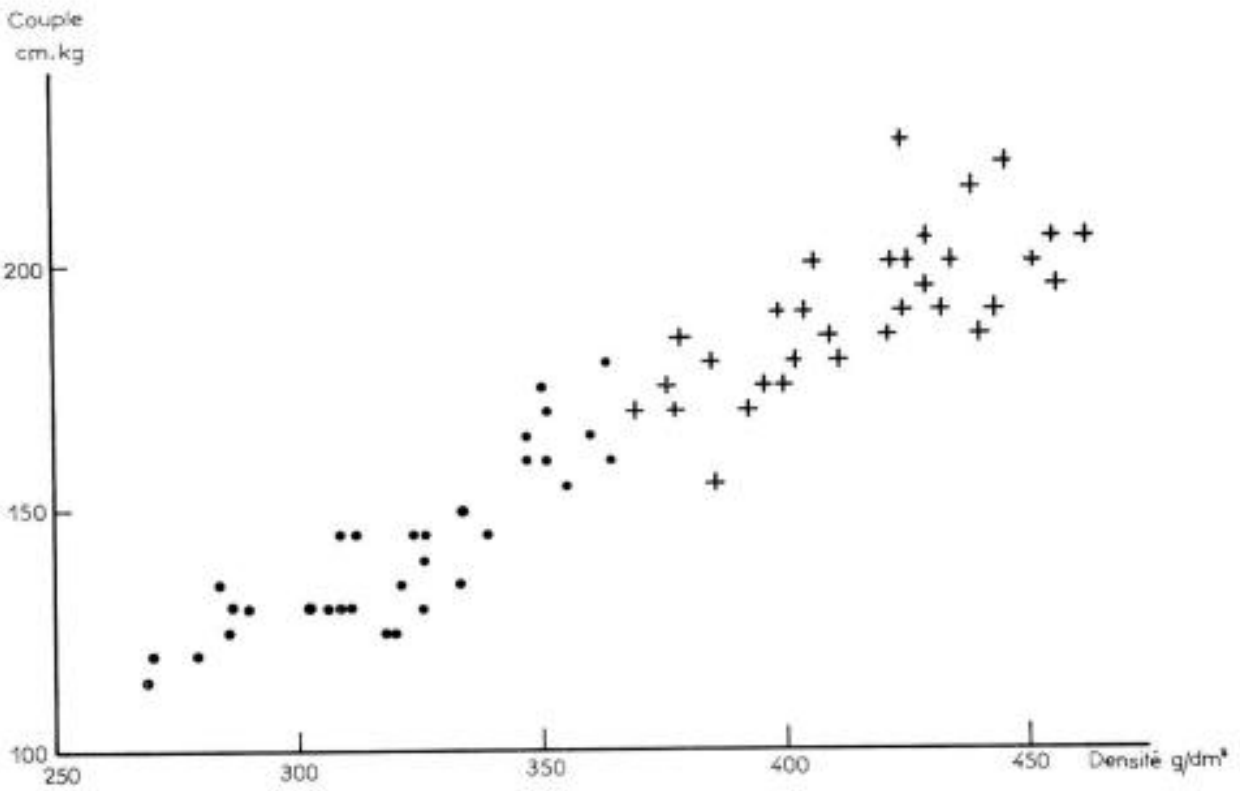

FIG. 3. - Influence de la densité du bois sur le couple de rorsion (modalité stabilisée superficielle) Fig. 3, - Effect of wood specific graviry on the torsion iorque for superficial measurement atter

- : Grandis ; + : Douglas. stabilization

Les densités sont exprimées en $\mathrm{g} / \mathrm{dm}^{3}$ et les couples de torsion en $\mathrm{cm} . \mathrm{kg}$, ces unités, non cohérentes entre elles, ayant été adoptées en raison de la possibilité qu'elles offrent de travailler sur des nombres entiers ayant de surcroit le même nombre de chiffres significatifs.

Dans chaque cas, les résultats ont été obtenus à partir de 64 paires de données, les analyses de régression ayant porté sur l'ensemble des mesures, tous arbres, tous opérateurs et toutes tarières réunis.

On doit tout d'abord noter la valeur extrêmement élevée des coefficients de corrélation $\left(^{*}\right)$; ils sont en particulier notablement plus forts que ceux trouvés précé-

( ) Voir à titre d’exemple la figure 3 sur laquelle sont reportées les variations du couple en fonction de la densité pour la modalité esuperficielle stabilisée ». 
demment, ce qui tient au fait que, pour la première fois, l'échantillon de densité a été strictement limité à la zone de bois comprimé lors de la mesure du couple de torsion.

Pour les mesures profondes, le couple est plus élevé alors que la densité du bois est plus faible que pour les mesures superficielles. On peut penser qu'une décompression du bois intervient au-delà des épaulements, entrainant un effet de freinage sur l'extérieur de la partie cylindrique de la tarière. En toute hypothèse, cette seule observation montre l'importance qu'il y a à mesurer les couples de torsion toujours à la même profondeur si l'on veut obtenir des valeurs qui soient comparables.

Aux deux niveaux étudiés, le couple moyen stabilisé est supérieur au couple démarrage, et il en était de même pour toutes les mesures individuelles sans exception : aucune explication ne peut actuellement être avancée pour rendre compte de ce phénomène.

Dans les deux cas également. les coefficients de corrélation densité-couple sont plus élevés quand la lecture est faite après stabilisation du torsiomètre. C'est là une information importante : le couple de torsion ne doit pas être lu au démarrage, mais au moment du palier qui se produit régulièrement après une rotation d'environ un quart de tour.

Pour le reste, les mesures profondes semblent présenter un double avantage: la liaison avec la densité est un peu plus étroite et surtout, à une même différence de densité, correspond un écart plus important dans la valeur du couple du couple de torsion; la sensibilité de la méthode est donc meilleure.

TABLEAU 2

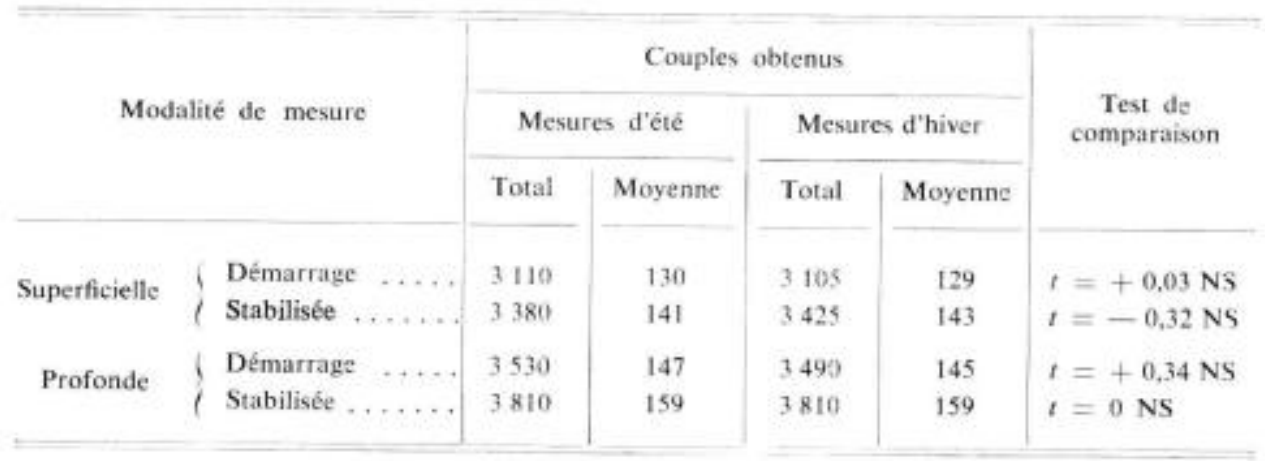

Modalité de mesure: method of measurement.

Couples obtenus : torsion torque values.

Mesures d'été: Summer measurement.

Mesures d'hiver: Winter measurement,

Total : total.

Moyenne : mean value.

Test de comparaison : test of comparison.

Démarrage superficiel : starting for shallow measurement.

Stabilisé superficiel: shallow measurement after stabilization of the torsiometer.

Stabilisé profond: deep measurement after stabilization.

Démarrage profond: starting for deep measurement. 
La modalité de mesure * stabilisée profonde s semble donc devoir être retenue, dans le cas général, pour les analyses de routine, mais sa supériorité sur la mesure * stabilisée superficielle s est faible, et il existe au moins un cas où celle-ci doit être préférée: il concerne les arbres de faible diamètre pour lesquels un enfoncement de la tarière au-delà du strict minimum indispensable risque de la mettre plus ou moins au contact d'une zone de bois juvénile non représentatif.

\subsection{2. - Influence de l'époque des mesures.}

Les résultats de la comparaison sont résumés dans le tableau 2 ci-contre: ils ont été obtenus à partir de 24 paires de mesures ( 3 opérateurs $\times 8$ arbres).

Quelle que soit la modalité de mesure, il n'y a pas de différence significative entre les couples de torsion relevés en sève et hors sève.

Les écarts maximaux entre les moyennes n'excèdent pas $2 \mathrm{~cm} . \mathrm{kg}$ et n'ont pas toujours le même signe.

La mesure ¿stabilisée profonde s parait une fois de plus la meilleure.

\section{3. - EXPERIENCE \& SIVRITE 2 s}

\section{1. - Matériel et méthodes}

Cette expérience a été entreprise peu de temps après la précédente sur 18 arbres: 9 Douglas et 9 Grandis, dans le but d'étudier l'effet \& affútage de la tarière * et leffet \& opérateur \$.

Dans cette série de mesures, deux tarières ont été utilisées dont d'une suivant deux modalités différentes. On a donc procédé à trois types de sondages correspondant aux références:

- tarière $\mathrm{n}^{\prime \prime} 1$ : tarière normale de $5,3 \mathrm{~mm}$ de diamètre parfaitement affûtée,

- tarière $\mathrm{n}^{\prime \prime}$ 2: même tarière, volontairement usée, après achèvement des sondages du type * tarière $\mathrm{n}^{-1} 1$ \%, par exécution de 300 sondages de $10 \mathrm{~cm}$ de longueur réalisés dans un bois dur (Hêtre), sans entretien intermédiaire,

- tarière $\mathrm{n}^{\circ} 3$ : autre tarière de $5,3 \mathrm{~mm}$ de diamètre fortement abîmée (crans de $2 \mathrm{~mm}$ de longueur et de $1,5 \mathrm{~mm}$ de profondeur sur le biseau terminal).

Les mesures ont été faites par trois opérateurs correspondant aux références $\mathrm{A}, \mathrm{B}$ et $\mathrm{C}$.

Chacun des 18 arbres a été sondé en 9 points différents répartis sur trois génératrices distantes de $7 \mathrm{~cm}$ et sur trois niveaux distants de $10 \mathrm{~cm}$ pour éviter les interactions entre trous voisins.

A chaque point avait été affecté un couple opérateur $x$ tarière suivant un dispositif en carré gréco-latin du type :

De cette façon chaque opérateur et chaque tarière ne figuraient qu'une seule fois dans chaque ligne et chaque colonne du dispositif.

En outre, les permutations nécessaires ont été opérées d'un arbre à l'autre pour que chacune des neuf combinaisons soit représentée une fois et une seule en chacune des neuf positions pour chaque essence prise séparément. 
La zone de sondage était situće à hauteur d'homme et choisie de façon à éviter la présence de næuds ou d'autres causes possibles danomalies, telles que des renflements anormaux du tronc.

On n'a pas tenu compte de lorientation du dispositif de sondage, si bien que lon peut considérer que sa position autour du tronc varie de façon aléatoire d'un arbre ì lautre.

Bien que les mesures profondes semblent devoir être préférées lors des analyses de rçutine comme l'a montré lexpérience * Sivrite 1 \%, on n’a procédé, dans le cas de * Sivrite 2 \%, qu'à la lecture des mesures relatives aux modalités \& démarrage superficiel et a stabilisé superficiel ». En effet, les 9 points des carrés gréco-latins étant choisis proches les uns des autres pour conserver le plus d'homogénéité possible à l'ensemble, il a paru préférable de s'abstenir de faire les lectures correspondant aux modalités profondes car. à chacun des niveaux, les trajets des tarières convergent vers laxe de l'arbre, et ces lectures risquaient de prendre en compte des interactions indésirables entre trous de sondage (voir figure 4).
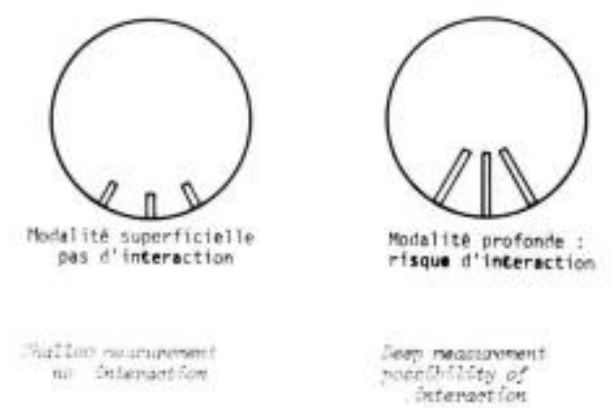

Fi6, 4. - Schéma montrant la raison pour laquelle on s'est limité à la modalité superficielle dans lexpérience e Sivrite 2 ?

F16. 4. - Diagram explaining why the deep tarques have not been measured in the experience \&ivrite 2 ,

\section{2. - Résultats}

Les résultats sont donnés pour chaque essence prise séparément, les Douglas représentant le cas d'un bois à densité élevée et les Grandis celui d'un bois à densité faible.

Le dispositif adopté n'est en réalité pas vraiment orthogonal, mais s'il n'isole pas toutes les interactions possibles, il permet cependant d'étudier les effets arbre, position dans l'arbre (effet colonne du carré gréco-latin), hauteur dans l'arbre (effet ligne), tarière, opérateur.

\subsection{1. - Facteurs annexes.}

Dans cette expérience, le but n’étant pas l'étude des variations du couple en fonction des facteurs \& arbre \$, "position dans l'arbre * et \& hauteur dans l'arbre *, mais seulement leur séparation des autres effets, nous dirons simplement que l'hétérogénéité des facteurs \& arbre * et "position dans l'arbre * est marquée aussi bien chez les Douglas que chez les Grandis, ce qui n'est pas surprenant quand on connait la variabilité de la densité du bois à la fois entre individus et à l'intérieur des individus. 
Il faut remarquer que ce dispositif ne conférait pas au facteur eposition dans l'arbre , une signification intrinsèque et l'analyse de variance a porté en réalité d'abord sur le facteur a arbre $\mathrm{x}$ position dans larbre *, un calcul simple permettant ensuite disoler le facteur eposition dans l'arbre s.

Le facteur \& hauteur dans l'arbre * a une signification propre. Chez le Douglas, il semble qu'il n'introduise pas d'hétérogénéité marquée entre les couples lus aux différents niveaux. Chez le Grandis, il apparaît plus souvent une différence entre les niveaux, notamment entre le plus haut et le plus bas qui portent des trous de sondage distants de $20 \mathrm{~cm}$; les couples mesurés au niveau le plus haut y sont plus faibles que ceux du niveau le plus bas.

\subsection{2. - Influence de l'état de la tarière.}

\subsection{1. - Cas du Douglas.}

Les résultats sont portés dans le tableau 3.

On peut tout d'abord noter que le couple moyen au démarrage est inférieur au couple moyen stabilisé d'environ $20 \mathrm{~cm}, \mathrm{~kg}$ pour chacune des trois tarières, comme dans l'expérience * Sivrite 1 \%.

Cependant, le fait le plus surprenant est que la tarière $\mathrm{n}^{\prime \prime} 1$ affûtée a conduit à des mesures de couples supérieures à celles de la tarière $\mathrm{n}^{\text {" }} 2$ qui était usée, la différence valant environ $8 \mathrm{~cm} . \mathrm{kg}$ au démarrage et $10 \mathrm{~cm} . \mathrm{kg}$ à l'état stabilisé.

La tarière $\mathrm{n}^{* 3}$, abîmée, a donné les couples les plus forts. Ainsi, chez le Douglas, les trois types de tarières ont fourni des résultats statistiquement différents. On pourrait donc craindre qu'à densité du bois égale, les valeurs lues au torsiomètre avec une tarière affûtée décroissent au fur et à mesure des prélèvements.

Mais il convient de noter le caractère anormal du traitement subi par la tarière en passant de l'état *tarière 1 s à l'état *tarière 2 s par la réalisation pratiquement ininterrompue d'un grand nombre de sondages dans un bois très dur. Il est certain que l'on a obtenu ainsi un polissage tout à fait exceptionnel de la tarière qui. en fin d'opération, brillait littéralement comme un miroir. Un tel état de choses ne se produit pas en utilisation normale quand les séries de sondage successives sont séparées les unes des autres par des intervalles au terme desquels l'état de surface du métal se retrouve sensiblement identique d'une opération à la suivante.

Quant aux différences relevées avec la tarière $n " 3$, elles ne sont absolument pas surprenantes, et l'on peut au contraire être étonné du faible écart qui la sépare des deux autres, compte tenu de son état de dégradation extrême. Au total, l'influence de l'affûtage paraît, sauf cas exceptionnel, négligeable. Ceci s'explique aisément, car si la qualité du biseau terminal intervient pour faciliter l'introduction de la tarière dans le bois, elle ne joue plus qu'un rôle très limité à partir du moment où elle s'enfonce sous l'effet de l'effort exercé sur les filets; l'essentiel de la résistance à vaincre est alors celle qui s'oppose à la compression du bois depuis le diamètre de la carotte jusqu'au diamètre du trou qui subsiste après prélèvement. c'est-à-dire dans le cas général, depuis $5 \mathrm{~mm}$ jusqu'à $8 \mathrm{~mm}$ environ. 
Tabl. 3. - Douglas. - Analyse de variance et comparaison de moyennes - Influence de létat de la tarière

TABI., 3. - Dougl.AS FIR. - Analysis of variance and comparison between mean values - Effect of borer maintenance

\begin{tabular}{|c|c|c|c|c|c|c|c|c|}
\hline \multirow{2}{*}{$\begin{array}{l}\text { Modalitá } \\
\text { de mesure }\end{array}$} & \multicolumn{3}{|c|}{ Coupl: m jyen en $\mathrm{cm} . \mathrm{kg}$} & \multicolumn{4}{|c|}{ Carré moyen } & \multirow{2}{*}{$\mathbf{F}_{3 w}^{2}$} \\
\hline & $\begin{array}{c}\text { tarière } \\
n^{\circ} \quad 1\end{array}$ & $\begin{array}{c}\text { tarière } \\
\mathrm{n}^{*} 2\end{array}$ & $\begin{array}{c}\text { tarière } \\
n^{\circ} 3\end{array}$ & Erreur & $\begin{array}{c}\text { Degré } \\
\text { de liberté }\end{array}$ & Traitement & $\begin{array}{c}\text { Degré } \\
\text { de liberté }\end{array}$ & \\
\hline Démarrage superficiel & 179,444 & 171,481 & 200,370 & 61,46 & 36 & 6011,47 & 2 & $97,82^{=184}$ \\
\hline Stabiliš́ superficiel & 201.665 & 190.740 & 217,963 & 65,16 & 36 & 5064,93 & 2 & $77,73 \div \neq \neq$ \\
\hline
\end{tabular}

Comparaison de moyennes:

Modalité de mesure :

tarièrz $\mathrm{n}^{*} 2$

171,481

190,740 tarière $\mathrm{n}^{*} 1$

179,444

201,666 tarière $\mathrm{n}^{\circ}-3$

200,370

217,963
Au seuil de signification de 1 is écart-type d'une différence: $2,135 \mathrm{~m} . \mathrm{kg}$

Au seuil de signification de 1 cm écart-type d'une différence: $2.198 \mathrm{~m} \cdot \mathrm{kg}$

Modalité de mesure: method of measurement.

Démarrage superficiel : starting for shallow measurement.

Stabilisé superficiel: shallow measurement after stabilization of the torsiometer.

Couple moyen : mean values of torsion torques.

Tarière : increment borer.

Carré moyen : mean squares.

Erreur : error.

Degré de liberté : degree of freedom.

Traitement : treatment.

Szuil de signification: significance level.

Ecart-type d'une différence : standard deviation of a difference. 


\subsection{2. - Cas du Grandis.}

Pour cette essence, dont le bois est bien moins dense que celui du Douglas, on lit des couples plus faibles, mais les mêmes phénomènes se répètent avec toutefois moins d'intensité (tableau 4).

Le couple moyen au démarrage est inférieur au couple moyen stabilisé d'environ $16 \mathrm{~cm}$. $\mathrm{kg}$. l'écart le plus faible étant observé avec la tarière $\mathrm{n}^{\prime \prime} 3$, comme dans le cas du Douglas.

De même, la tarière $\mathrm{n}^{\prime \prime} 1$ donne des valeurs de couples supérieures à celle de la tarière $\mathrm{n}^{*} 2$, mais la différence n'est plus significative; elle ne vaut plus que $3 \mathrm{~cm} . \mathrm{kg}$ au démarrage ou à l'état stabilisé.

La tarière $\mathrm{n}^{\circ} 3$, elle, présente toujours des différences très significatives avec les deux autres.

Il semblerait ainsi que, pour une essence dont le bois a une faible densité, l'action combinée du polissage et de l'usure de la tarière au cours d'une série de prélèvements présente moins d'inconvénients que dans le cas d'une essence à forte densité.

\subsection{3. - Influence de lopérateur.}

Pour vérifier s'il existait un facteur personnel dans l'emploi du torsiomètre, on a fait faire les mesures par trois opérateurs différents $\mathrm{A}, \mathrm{B}$ et $\mathrm{C}$ qui effectuèrent indépendamment les uns des autres la série de sondages aux emplacements prévus dans le carré gréco-latin, et les valeurs des couples lus au démarrage et à l'état stabilisé furent annoncées par chacun d'eux sans connaître les résultats trouvés par les deux autres.

\subsection{1. - Cas du Douglas (voir tableau 5).}

L'analyse de variance montre que, pour la modalité \& démarrage superficiel \$, il existe, dans cette expérience, une hétérogénéité dans les résultats trouvés par les opérateurs A, B et C ; l'écart maximal s'observe entre A et C et vaut $6 \mathrm{~cm}$. $\mathrm{kg}$, soit $3,3 \%$ de la valeur mesurée.

Si les mesures sont faites à l'état * stabilisé superficiel », on ne peut plus mettre en évidence de différences entre les trois opérateurs car le test $\mathrm{F}$ n'est plus significatif, et l'écart maximal entre $\mathrm{A}$ et $\mathrm{C}$ ne vaut plus que $4 \mathrm{~cm}, \mathrm{~kg}$, soit $2 \%$ de la valeur mesurée.

\subsection{2. - Cas du Grandis.}

Dans les deux modalités, le test $\mathrm{F}$ est significatif ; il existe une hétérogénéité plus marquée au démarrage où l'écart maximal atteint $10 \mathrm{~cm} . \mathrm{kg}$, soit $8.5 \%$ de la valeur mesuré, qu’à l'état stabilisé où il s'abaisse à $7 \mathrm{~cm}$. kg. soit $5 \%$ de la valeur mesurée (tableau 6).

Il semble bien que l'influence du facteur personnel n'est pas négligeable, mais elle est moins marquée lorsque les lectures sont faites suivant la modalité \& stabilisée superficielle s.

Deux opérateurs $\mathrm{B}$ et $\mathrm{C}$ ont donné des résultats voisins, tandis que $\mathrm{A}$ a fait en général des lectures inférieures au groupe B et C. L'emploi du torsiomètre par 
TABr. 4. - Grandis. - Analyse de variance et comparaison de moyennes - Influence de létat de la tarière

TABL. 4. - GRAND FIR. - Annalysis of variance and comparison between mean values - Etfect of borer maintenance

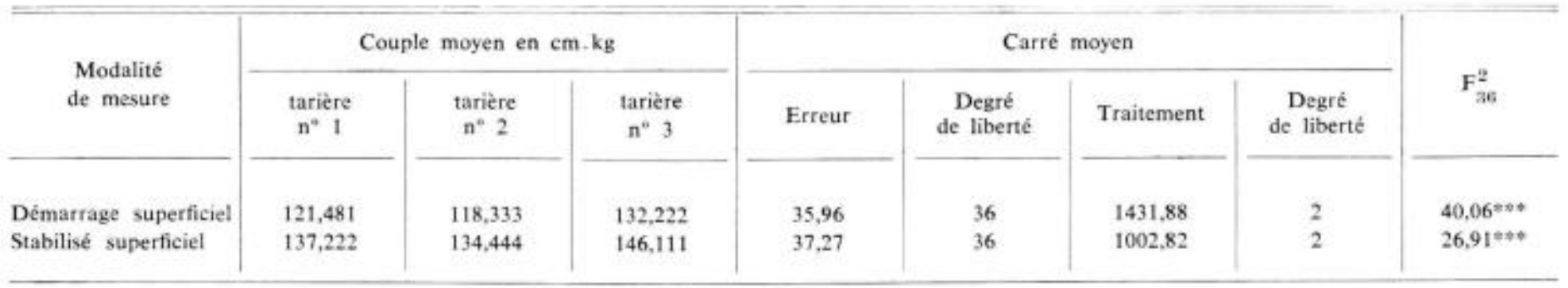

Comparaison de moyennes:

Modalitế de mesure

Démarrage superficiel ......

$\begin{array}{cc}\text { tarière } n^{*} 2 & \text { tarière } n^{*} 1 \\ 118,333 & 121,481\end{array}$

tarière $\mathrm{n}^{\mathrm{*}} 3$

132,222

Au seuil de signification de 1 co écart-type d'une différence : $1,630 \mathrm{~m} \cdot \mathrm{kg}$

Stabilisé superficiel

$134,444 \quad 137,222$

146,111

Au seuil de signification de 1 tee écart-type d'une différence: $1.662 \mathrm{~m} . \mathrm{kg}$

Modalité de mesurz: method of measurement.

Démarrage superficiel : starting for shallow measurement.

Stabilisé superficiel: shallow measurement after stabilization of the torsiometer.

Couple moyen : mean values of torsion torques.

Tarière : increment borer.

Carré moyan; mean squares.

Erreur : error.

Degré de liberté : degree of freedom.

Traitement : treatment.

Seuil de signification: significance level.

Ecart-type d'une différence : standard deviation of a difference. 
TABL. 5. - Dougl.as. - Analyse de variance et comparaison de moyennes - Influence de lopérateur

Tarl. 5. - Douglas FIR. - Analysis of variance and comparison between mean values - Effect of measurer

\begin{tabular}{|c|c|c|c|c|c|c|c|c|}
\hline \multirow{2}{*}{$\begin{array}{l}\text { Modalité } \\
\text { de mesure }\end{array}$} & \multicolumn{3}{|c|}{ Couple moyen en $\mathrm{cm} \cdot \mathrm{kg}$} & \multicolumn{4}{|c|}{ Carré moyen } & \multirow{2}{*}{$\mathrm{F}^{2}$} \\
\hline & Opérateur A & Opérateur B & Opérateur C & Erreur & $\begin{array}{c}\text { Degré } \\
\text { de liberté }\end{array}$ & Traitement & $\begin{array}{c}\text { Degré } \\
\text { de liberté }\end{array}$ & \\
\hline Démarrage superficiel & 180,370 & 186,481 & 184,444 & 61,46 & 36 & 378,73 & 2 & 6,16 की \\
\hline Stabilisé superficiel. & 201,666 & 205,555 & 203,148 & 65,16 & 36 & 104,02 & 2 & $1,60 \mathrm{NS}$ \\
\hline
\end{tabular}

Comparaison de moyennes:

Modalité de mesure

Démarrage superficiel ......

\begin{tabular}{|c|c|c|}
\hline $\begin{array}{c}\text { Opérateur A } \\
180,370\end{array}$ & $\begin{array}{c}\text { Opérateur C } \\
184,444\end{array}$ & $\begin{array}{c}\text { Opérateur B } \\
186,481\end{array}$ \\
\hline & & \\
\hline Opérateur A & Opérateur C & Opérateur B \\
\hline 201,666 & 203,148 & 205,555 \\
\hline
\end{tabular}

Au seuil de signification de $1 \%$

Au seuil de signification de 1 \%

Ecart-type d'une différence : $2,135 \mathrm{~m} \cdot \mathrm{kg}$

Stabilisé superficiel

201,666

203,148

Pas de différences entre opérateurs

Ecart-type d'une différence : $2,198 \mathrm{~m} . \mathrm{kg}$

Modalité de mesure: method of measurement.

Démarrage superficiel: starting for shallow measurement.

Stabilisé superficiel : shallow measurement after stabilization of the torsiometer.

Couple moyen: mean values of torsion torques.

Opérateur : measurer.

Carré moyen: mean squares.

Erreur : error

Degré de libertá: degree of freedom.

Traitement : treatment.

Seuil de signification : significance level.

Ecart-type d'une différence : standard deviation of a difference.

Pas de différences entre opérateurs: no difference between measurers. 
TaBl. 6. - Grandis. - Analyse de variance et comparaison de moyennes - Influence de Topérateur

TABL. 6. - Grand FIR. - Analysis of variance and comparison berween mean values - Effect of measurer

\begin{tabular}{|c|c|c|c|c|c|c|c|c|}
\hline \multirow{2}{*}{ Modalité de mesurz } & \multicolumn{3}{|c|}{ Couple moyen en $\mathrm{cm} . \mathrm{kg}$} & \multicolumn{4}{|c|}{ Carré moyen } & \multirow[b]{2}{*}{$\mathbf{F}_{; 6 !}^{2}$} \\
\hline & Opérateur A & Opérateur B & Opśrateur C & Erreur & $\begin{array}{l}\text { Dagré } \\
\text { de liberté }\end{array}$ & Traitement & $\begin{array}{c}\text { Degré } \\
\text { de liberté }\end{array}$ & \\
\hline Démarrage superficiel & 117,592 & 127,037 & 127.407 & 35,96 & 36 & 881,43 & 2 & $24,36^{206}$ \\
\hline Stabilisé superficiel . & 135,556 & 139,629 & 142.592 & 37,27 & 36 & 337,03 & 2 & $9,04^{004}$ \\
\hline
\end{tabular}

Comparaison de moyennes:

Modalité de mesure

\begin{tabular}{ccc}
$\begin{array}{c}\text { Opérateur A } \\
117.592\end{array}$ & $\begin{array}{r}\text { Opérateur B } \\
127,037\end{array}$ & $\begin{array}{r}\text { Opérateur C } \\
127.407\end{array}$ \\
\hline \multirow{2}{*}{135.555} & 139,629 & 142.592 \\
\hline
\end{tabular}

Au seuil de signification de 1 \%e ếcart-type d'une différence : $1,630 \mathrm{~m} \cdot \mathrm{kg}$

Stabilisé superficiel iciel....

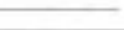

Au seuil de signification de $5 \%$

Au seuil de signification de $1 \%$ ecart-type d'une différence: $1,662 \mathrm{~m} . \mathrm{kg}$

Modalité de mesur2: method of measurement.

Démarrage superficiel: starting for shallow measurement.

Stabilisé superficiel : shallow measurement after stabilization of the torsiometer.

Couple moyen: mean values of torsion torques.

Opérateur: measurer.

Carré moyen: mean squares.

Erreur : error.

Degré de liberté: degree of freedom.

Traitement : treatment.

Ecart-type d'une différence: standard deviation of a difference,

Seuil de signification: significance level, 
différentes personnes semble faire apparaître dans certains cas des différences systématiques dont il pourrait être facile de se débarrasser en appliquant aux résultats de chaque opérateur un coefficient approprié. En outre, des essais ultérieurs ont montré que ce facteur individuel était lié à la vitesse avec laquelle était actionné le manche du torsiomètre, et qu'il disparaissait lorsque les modalités de mise en cuvre de l'appareil étaient rendues suffisamment uniformes.

\section{4. - EXPERIENCE * SAINT AVOLD *}

La valeur extrêmement élevée des coefficients de corrélation trouvés entre densité et couple de torsion ne manque pas de surprendre: on n'en obtient jamais de pareils lorsqu'on étudie sur éprouvettes normalisées la liaison entre la densité et l'une quelconque des caractéristiques mécaniques.

Les explications possibles sont au nombre de deux :

- dans l'arbre vivant, la tarière comprime un bois qui est saturé d'eau, alors que les essais mécaniques classiques sont faits sur des échantillons à l'état sec à l'air,

- la contrainte exercée par la tarière présente un caractère complexe, et la résistance à l'avancement qui en résulte pourrait être plus liée à la densité que ne le sont les diverses caractéristiques mécaniques prises isolément.

\section{1. - Matériel et méthode}

Pour vérifier cette double hypothèse, lexpérience suivante a été entreprise :

32 Epicéas ont été sondés à hauteur d'homme, et deux mesures stabilisées du couple de torsion faites sur chacun d'eux: T'une au moment où les épaulements de la tariêre disparaissant dans le bois, l'autre après trois tours da tarière, soit $37,5 \mathrm{~mm}$ plus loin.

La densité du bois a été déterminée par la méthode de saturation intégrale sur les fragments de carottes correspondant à ces deux niveaux.

Des éprouvettes normalisćes ont été façonnées au voisinage immédiat des trous de sondage et à des distances de léeorce approximativement égales à celles des deux mesures faites au torsiomètre (voir schéma de la figure 5).

On a ainsi obtenu pour chaque arbre 8 éprouvettes (4 cốté écorce, numérotées de 1 à 4 , 4 côté moelle, numérotées de 5 à 8 ) ; les éprouvettes situées dans le même plan longitudinal radial que le trou de sondage (éprouvettes de numéro pair) ont servi à des essais en compression axiale, celles disposées latéralement par rapport à la carotte (numéros impairs) à des essais en compression de flane. On avait de la sorte à chaque niveau deux éprouvettes analogues dont l'une, choisie au hasard, a été essayée à l'état frais, et l'autre à l'état sec à l'air.

Pour tenir compte du retrait, les dimensions initiales des échantillons étaient de $21.5 \mathrm{~mm}$ dans le sens tangentiel, 21 dans le sens radial, et 60 dans le sens longitudinal, de façon à aboutir à des dimensions à l'état see à l'air voisines de celles des éprouvettes normalisées classiques $(2 \times 2 \times 6 \mathrm{~cm})$.

Les essais en compression axiale ont été faits en se conformant à la norme NF 51.007.

Pour la compression de flanc, on a déterminé la charge nécessaire pour réduire de un tiers la dimension de l'éprouvette dans le sers radial: un micro-contact, réglé sur la dimensıon finale à obtenir, avait été mis en place sur la presse, interrompant leffort de compression lorsque la limite fixée était atteinte. 

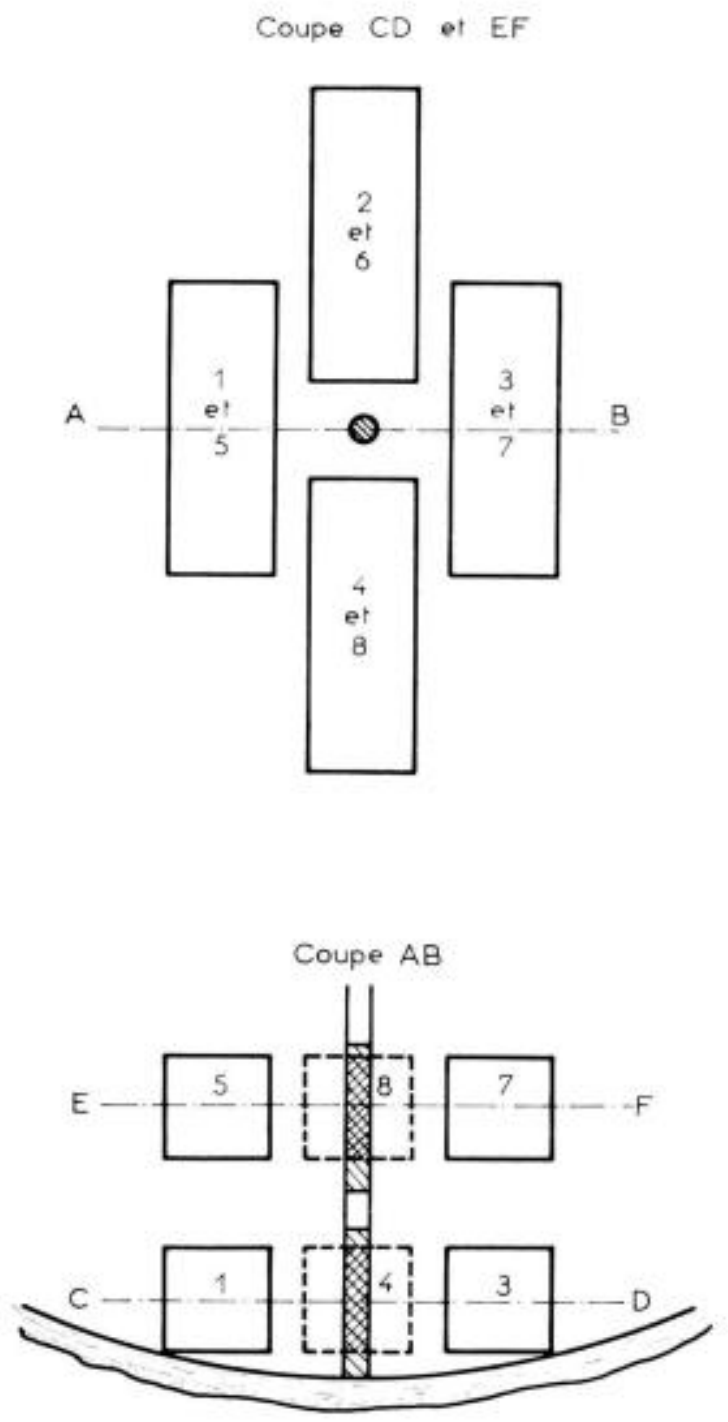

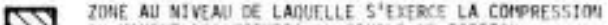
AII WMAEMT DES RESLRES DII COUPLE TE TORSIOH

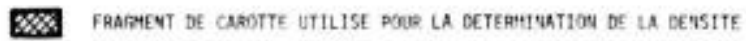

Echelle: $\longrightarrow 1 \mathrm{~cm}$

F1G. 5. - Schéma montrant la disposition relarive des trous de sondage et des éprouvettes normalisées dans Texpérience \& $S t \cdot A$ vold s

F16. 5. - Diagram showing the relative situation of the boring and of the standardized samples in the experience \& St-Avolds 
En outre, à $23 \mathrm{~cm}$ au-dessous du trou de tarière (distance relativement importante due au débit dans l'intervalle d'autres éprouvettes destinées à des études de rétractibilité). mais sur la même génératrice, ont été façonnés deux échantillons parallélépipédiques (l'un côté moelle, l'autre côté écorce), de $8 \mathrm{~cm}$ dans les directions tangentielle et axiale, et de $2 \mathrm{~cm}$ dans le sens radial, devant servir à des essais de dureté. Pour ceux-ci, on pouvait penser à priori que la méthode à la bille serait la plus intéressante à essayer en raison des analogies que lon retrouve avec la pénétration de la tarière qui. comme la bille, exerce des contraintes à la fois dans le sens axial et dans le sens tangentiel ; on a done déterminé la charge nécessaire pour enfoncer en direction radiale, jusqu’à son plan diamétral, une bille d'acier ayant une section de $1 \mathrm{~cm}^{2}$ (méthode Janka).

Sur l'éprouvette de $8 \times 8 \times 2 \mathrm{~cm}$ décrite plus haut, ont été faites deux déterminations de dureté, l'une à l'état frais, l'autre à l'état sec, les points de mesure étant situés sur deux génératrices différentes et suffisamment éloignées des bords de l'éprouvette pour qu'il n'y ait ni interaction ni effet de bordure.

Malheureusement, il s'est avêré que 10 des arbres devant faire l'objet de l'expérience avaient été abattus prématurément, en sorte que leur bois était partiellement desséché : en conséquence, toutes les études concernant l'état saturé n'ont pu être faite que sur un maximum de 22 arbres; en outre, un tout petit nombre d'éprouvettes comportait des nceuds, ce qui a réduit légèrement le nombre d'échantillons disponibles pour certains essais.

Les bois retenus pour les essais à létat frais ont été conservés dans l'eau entre l'arrivée au laboratoire et le débit, et il en a été de même pour les éprouvettes entre le débit et les essais.

\section{2. - Résultats}

\subsection{1. - Corrélations entre densité, couple de torsion et caractéristiques mécaniques.}

L'ensemble des résultats est consigné dans le tableau 7.

Les divers coefficients de corrélation entre la densité du bois, le couple de torsion mesuré lors du prélèvement des carottes et les diverses caractéristiques mécaniques déterminées sur éprouvettes parallélépipédiques, soit à l'état saturé, soit à l'état sec à l'air, sont reportés sur les tableaux 8 et 9 (sont indiqués entre parenthèses dans chaque cas le nombre de comparaisons à partir desquelles les coefficients de corrélation ont êté calculés).

On peut faire les observations suivantes :

- Les liaisons entre couple et densité, bien que très significatives, sont plus faibles que dans l'expérience * Sivrite 1 ; ; ceci tient vraisemblablement au fait que les fragments de carottes ayant servi aux déterminations de densité ont été limités à une longueur de $2 \mathrm{~cm}$ correspondant à la dimension radiale des éprouvettes normalisées; ils représentent done des zones moins étendues que celles sur lesquelles s'exerçait l'effort de compression lors des mesures du couple.

- Les résultats relatifs aux échantillons côté écorce, d’une part, et côté moelle de l'autre, sont dans l'ensemble assez concordants; on doit noter en particulier dans les deux cas l'absence de liaison significative entre la compression axiale à l'état sec et les diverses autres caractéristiques mécaniques, que celles-ci aient été déterminées sur éprouvettes ou par mesure au torsiomètre sur les arbres vivants. La seule différence importante concerne le coefficient de corrélation entre la dureté à l'état sec et la compression de flanc à l'état frais qui est très significatif côté moelle, et non significatif (mais voisin du seuil de signification), côté écorce. 
TABLEAU 7

\begin{tabular}{|c|c|c|c|c|c|c|c|c|c|c|c|c|c|c|c|c|}
\hline \multicolumn{9}{|c|}{ Echantillons côté écorce } & \multicolumn{8}{|c|}{ Echantillons côté moelle } \\
\hline \multirow{3}{*}{$\mathbf{N}^{\circ}$} & \multirow{3}{*}{$\begin{array}{l}\text { Den- } \\
\text { sité }\end{array}$} & \multirow{3}{*}{ Couple } & \multicolumn{3}{|c|}{ Etat saturé } & \multicolumn{3}{|c|}{ Etat sec à l'air } & \multirow{3}{*}{$\begin{array}{l}\text { Den- } \\
\text { sité }\end{array}$} & \multirow{3}{*}{ Couple } & \multicolumn{3}{|c|}{ Etat saturé } & \multicolumn{3}{|c|}{ Etat sec à l'air } \\
\hline & & & \multirow{2}{*}{ Dureté } & \multicolumn{2}{|c|}{ Compression } & \multirow{2}{*}{ Dureté } & \multicolumn{2}{|c|}{ Compression } & & & \multirow{2}{*}{ Dureté } & \multicolumn{2}{|c|}{ Compression } & \multirow{2}{*}{ Durstó } & \multicolumn{2}{|c|}{ Compression } \\
\hline & & & & de flane & axiale & & de flane & axiale & & & & de flanc & axiale & & de flane & axiale \\
\hline 1 & 438 & & & & & 660 & 1460 & 1940 & 345 & & & & & 315 & 750 & 1820 \\
\hline 2 & 431 & & & & & 690 & 1170 & 2410 & 345 & & & & & 490 & 1480 & 1690 \\
\hline 3 & 448 & & & & & 700 & 1170 & 2410 & 340 & & & & & 400 & 810 & 1700 \\
\hline 4 & 422 & & & & & 530 & 2060 & 1910 & 408 & & & & & 710 & 2450 & 2040 \\
\hline 5 & 386 & & & & & 525 & 1660 & & 400 & & & & & 525 & 1450 & 1940 \\
\hline 6 & 410 & & & & & 475 & 1020 & 1899 & 390 & & & & & 590 & 1280 & I 840 \\
\hline 7 & 410 & & & & & 525 & 1710 & 1810 & 391 & & & & & 590 & 990 & \\
\hline 8 & 402 & & & & & 500 & 910 & 2140 & 376 & & & & & 6.45 & 920 & 2080 \\
\hline 9 & 408 & & & & & 510 & 1280 & 2280 & 382 & & & & & 700 & 1110 & 2060 \\
\hline 10 & 395 & & & & & 625 & 1110 & 2290 & 363 & & & & & $\$ 10$ & 980 & 1970 \\
\hline 11 & 366 & 130 & 345 & 530 & 820 & 585 & 1330 & 1780 & 430 & 180 & 480 & & 1130 & 745 & 1740 & 2120 \\
\hline 12 & 361 & 115 & 385 & 650 & 870 & 535 & 880 & 1870 & 448 & 185 & 435 & 960 & 1200 & 715 & 1980 & 2350 \\
\hline 13 & 376 & 135 & 430 & 610 & 990 & 580 & 870 & 2180 & 400 & 175 & 380 & 560 & 1150 & 580 & 1080 & 2140 \\
\hline 14 & 399 & 135 & 325 & 810 & 1065 & 390 & 840 & 2150 & 402 & 165 & 325 & 860 & 1080 & 490 & 1070 & 2170 \\
\hline 15 & 391 & 140 & 440 & 640 & 970 & 550 & 1090 & 2080 & 392 & 170 & 470 & $\$ 10$ & 1030 & 685 & 880 & 1950 \\
\hline 16 & 407 & 140 & 385 & 580 & 1120 & 400 & 1010 & $\sum 050$ & 414 & 190 & 370 & 670 & 750 & 590 & 750 & 1810 \\
\hline 17 & 384 & 135 & 395 & 620 & 1010 & 530 & 1020 & 2030 & 389 & 165 & 425 & 770 & 1000 & 570 & 770 & 1850 \\
\hline 18 & 389 & 150 & 420 & 715 & 1100 & 620 & 930 & & 375 & 185 & 460 & 750 & & 500 & 770 & 1930 \\
\hline 19 & 394 & 135 & 340 & 515 & 985 & 410 & 940 & 2200 & 378 & 165 & 420 & 580 & 1040 & 425 & 670 & 1890 \\
\hline 20 & 395 & 130 & 355 & 680 & 1040 & 550 & 930 & 2210 & 396 & 180 & 460 & 570 & 1010 & 555 & 800 & 1810 \\
\hline 21 & 340 & 120 & 355 & & 780 & 355 & 920 & 1460 & 400 & 170 & 405 & 680 & 1000 & 385 & 790 & 2000 \\
\hline 22 & 362 & 120 & 395 & 710 & 890 & 430 & 770 & 1980 & 429 & 180 & 305 & 540 & 900 & 450 & 1160 & 2080 \\
\hline 23 & 367 & 120 & 315 & 400 & 960 & 600 & 990 & 1700 & 393 & 165 & 405 & 560 & 940 & 570 & 640 & 2000 \\
\hline 24 & 369 & 120 & 350 & 540 & 1010 & 525 & 940 & 2140 & 398 & 160 & 380 & 560 & 1000 & 530 & 630 & 2050 \\
\hline 25 & 391 & 125 & 325 & 550 & 940 & 435 & 850 & 1920 & 405 & 175 & 385 & 620 & 820 & 485 & 920 & 2160 \\
\hline 26 & 373 & 125 & 280 & 480 & 880 & 500 & 870 & 1.990 & 400 & 170 & 330 & 480 & 980 & 620 & 760 & 1690 \\
\hline 27 & 373 & 115 & 245 & 540 & 960 & 435 & 750 & 1930 & 386 & 165 & 385 & 540 & 1070 & 495 & 970 & 1870 \\
\hline 28 & 369 & 120 & 310 & 410 & 930 & 430 & 890 & 2050 & 374 & 165 & 335 & 580 & 980 & 550 & 980 & 2110 \\
\hline 29 & 361 & 125 & 335 & 440 & 960 & 385 & 780 & 2040 & 397 & 165 & 410 & 510 & 1010 & 560 & 550 & 1900 \\
\hline 30 & 373 & 135 & 410 & 640 & 790 & 650 & 1150 & 1710 & 375 & 185 & 475 & 820 & 770 & 800 & 1630 & 1560 \\
\hline 31 & 424 & 180 & 590 & 1000 & 1210 & 575 & 2140 & 2070 & 461 & 250 & 590 & 1300 & 1340 & 650 & 2590 & 1990 \\
\hline 32 & 444 & 180 & 635 & 1200 & 1200 & 670 & 2260 & 2010 & 488 & 280 & 800 & 1620 & 1375 & 840 & 2920 & 2050 \\
\hline
\end{tabular}

Les unités sont des $\mathrm{g} / \mathrm{dm}^{3}$ pour la densité,

Etat sec à l'air : dry conditions.

des $\mathrm{cm} / \mathrm{kg}$ pour le couple de torsion,

Densité: specific gravity (grams per cubic decimeter).

des $\mathrm{kg}$ pour la dureté (sur une section de $1 \mathrm{~cm}^{2}$ ),

des $\mathrm{kg}$ pour la compression de flanc (sur une section de $12 \mathrm{~cm}^{2}$ ).

Couple: torsion torque (centimeter $\times$ kilograms).

des $\mathrm{kg}$ pour la compression axiale (sur une section de $4 \mathrm{~cm}^{2}$ ).

Dureté : hardness (kilogram for a section of 1 square centimeter).

Echantillons côté écorce: shallow samples near bark.

Compression de flanc: crushing strength perpendicularly to the grain Echantillons côté moelle : deep samples.

direction (kilogram for a section of 12 square centimeters).

Compression axiale: crushing strengh parallelly to the grain direction 
TABL. 8. - Echantillons côté écorce - TABLE 8. - Shallow samples near bark

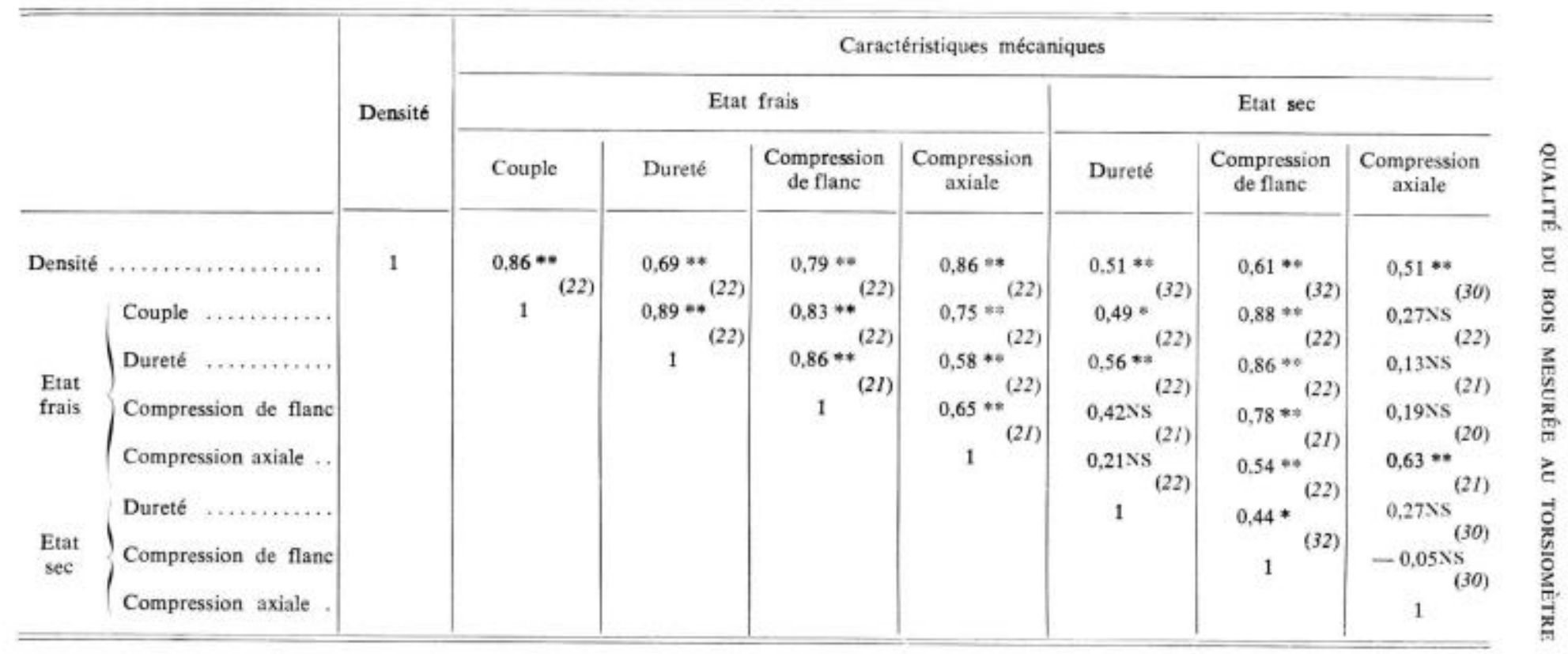

Caractéristiques mécaniques: mechanical characteristics.

Compression de flanc: crushing strength perpendicularly to the grain direction.

Compression axiale : crushing strength parallelly to the grain direction.
Densité : specific gravity Couple: torsion torque.

Dureté : hardness.

Etat frais: wet conditions.

Etat sec : dry conditions. 
тABL. 9. - Echantillons coté moelle - тAвLE 9. - Deep samples

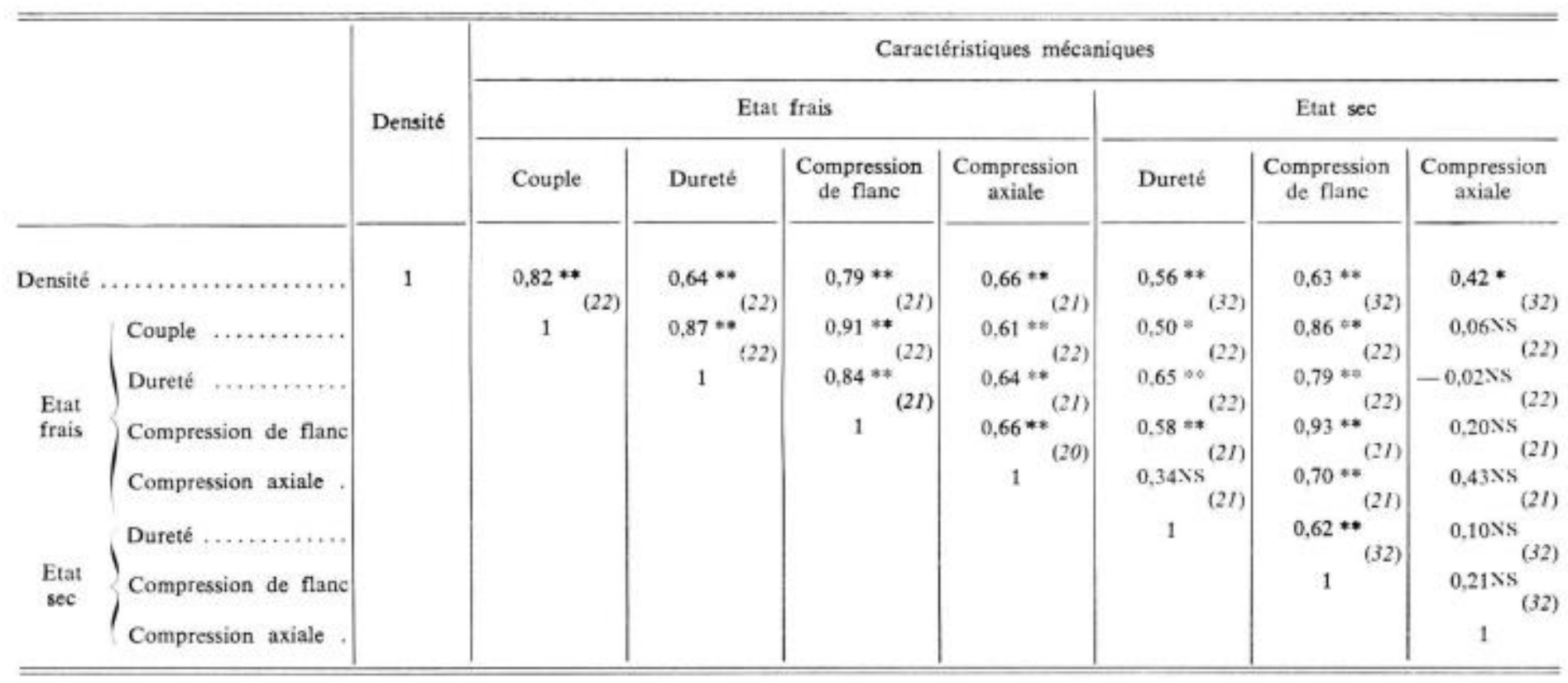

Caractéristiques mécaniques: mechanical characteristics.

Compression de flanc: crushing strength perpendicularly to the grain direction.

Compression axiale : crushing strength parallelly to the grain direction.
Densité : specific gravity

Couple : torsion torque.

Dureté : hardness.

Etat frais: wet conditions.

Etat sec: dry conditions. 
- Pour tous les échantillons, la densité est davantage liée aux caractéristiques mécaniques à l'état frais qu'aux mêmes critères de résistance mesurés sur des éprouvettes à l'état sec à l'air, ce qui confirme la première des hypothèses évoquées plus haut pour expliquer l'étroitesse de la relation qui lie le couple de torsion à la densité.

- Dureté, compression de flanc et compression axiale sont très corrélées entre elles à l'état saturé et, au contraire, beaucoup moins liées les unes aux autres à l'état sec.

- Dans les comparaisons entre mêmes caractéristiques mécaniques aux deux états de siccité étudiés, c’est la compression de flanc qui fournit les coefficients de corrélation les plus élevés, qu'il s'agisse des échantillons côté moelle ou des échantillons côté écorce.

\subsection{2, - Analyse de régression progressive.}

\subsection{1. - Explication de la densité.}

Il s'agit maintenant de vérifier l'autre hypothèse formuléc au départ, suivant laquelle la densité du bois pourrait être davantage liée à un ensemble de caractéristiques mécaniques qu'à l'une d'elles prise isolément.

L'analyse de régression progressive est un outil particulièrement bien adapté pour ce genre d'étude puisqu'on introduit à chaque palier la variable explicative qui apporte la meilleure information supplémentaire après celle au niveau précédent, c'est-à-dire que l'on ne tient pas compte des liaisons entre variables explicatives qui, ici, sont assez étroites.

Les équations des régressions sont données ci-dessous en clair, en utilisant les abréviations suivantes :

$\left.\begin{array}{l}\text { Dens }=\text { Densité en } \mathrm{g} / \mathrm{dm}^{3} \\ \text { Dur }=\text { Dureté en } \mathrm{kg} \\ \mathrm{CAx}=\text { Compression axiale en } \mathrm{kg} \\ \mathrm{CF}=\text { Compression de flanc en } \mathrm{kg}\end{array}\right\}$ définies comme indiqué plus haut.

Dans chaque cas, on ne mentionne que les équations significatives, c'est-à-dire que l'on s'est arrêté lorsque le palier suivant n'apportait aucune information supplémentaire elle-même significative.

Chaque équation est suivie du signe conventionnel indiauant le seuil de signification apportée $\left({ }^{\circ}=\right.$ seuil de $1 \%{ }^{*}=$ seuil de $\left.5 \%\right)$ et du coefficient de corrélation, simple au premier palier, multiple aux suivants.

Les coefficients de corrélation simple sont parfois légèrement différents de ceux qui figurent sur les tableaux 1 et 2 ; la raison en est que ceux-ci ont été déterminés un à un à l'aide d'un petit calculateur électronique de bureau, ce qui a permis d'utiliser, dans chaque cas, le maximum de couples de données disponibles; les analyses de régression, au contraire, ont été faites sur ordinateur en rendant orthogonal le disposítif, c'est-à-dire en éliminant totalement les échantillons pour lesquels une seule des six données concernant les caractéristiques mécaniques faisait défaut : le nombre de degrés de liberté était ainsi inférieur de 1, et quelquefois de 2 à celui dont on disposait pour les calculs de corrélation précédents. 
4.2211. - Exp'ication par caractéristiques mécaniques déterminées sur échantillons saturés.

Côté écorce :

$$
\begin{array}{ll}
\text { Dens }=224+0,162 \mathrm{CAx} & \mathrm{R}=0,829 * * \\
\text { Dens }=247+0,106 \mathrm{CAx}+0,050 \mathrm{CF} 1 & \mathrm{R}=0,895 * *
\end{array}
$$

Côté moelle :

Dens $=347+0.082 \mathrm{CFI}$

$$
\mathbf{R}=0,814 * *
$$

4.2212. - Explication par caractéristiques mécaniques déterminées sur échantillons secs.

Côté écorce :

$$
\begin{array}{ll}
\text { Dens }=343+0,043 \mathrm{CFI} & \mathrm{R}=0,641 * * \\
\text { Dens }=200+0,045 \mathrm{CFI}+0,070 \mathrm{CAx} & \mathrm{R}=0,838 *
\end{array}
$$

Côté moelle :

$$
\begin{array}{ll}
\text { Dens }=357+0,033 \mathrm{CF} 1 & \mathrm{R}=0,631 \text { ** } \\
\text { Dens }=223+0,029 \mathrm{CF} 1+0,071 \mathrm{CAx} & \mathrm{R}=0,732 \text { ** }
\end{array}
$$

Ainsi qu'on l'avait supposé, la prise en considération de deux caractéristiques mécaniques au lieu d'une améliore, dans trois cas sur quatre, de façon très nette l'explication de la densité. Par contre, jamais l'apport de la troisième variable explicative ne s'est avéré significatif (ce qui tient sans doute aux liaisons entre variables).

En régression progressive, comme en corrélation simple, les liaisons sont plus étroites avec les échantillons saturés qu'avec les secs. On doit observer en particulier que l'ensemble de la compression axiale et de la compression de flanc mesurées sur échantillons saturés côté écorce explique $80 \%$ des variations de la densité, ce qui, tout en étant supérieur, n'est pas très différent du pourcentage d'explication fourni par le couple de torsion.

\subsection{2. - Explication du couple de torsion.}

Ayant vérifié que, même en utilisant des éprouvettes parallélépipédiques normalisées, on pouvait obtenir d'excellentes liaisons avec la densité, à condition d'opérer sur des échantillons à l'état saturé, il était intéressant de voir si le couple de torsion était lui aussi en corrélation très étroite avec les caractéristiques mécaniques déterminées sur de tels échantillons.

Une analyse de régression progressive a permis de s'en assurer. On a obtenu: Côté écorce :

$$
\begin{array}{ll}
\text { Couple }=68+0,346 \text { Dur } & R=0,889 * * \\
\text { Couple }=29+0,267 \text { Dur }+0,054 \text { CAx } & R=0,929 * *
\end{array}
$$

Côté moelle :

$$
\begin{array}{ll}
\text { Couple }=114+0,094 \mathrm{CF} 1 & \mathbf{R}=0,906^{* *} \\
\text { Couple }=92+0,060 \mathrm{CF} 1+0,215 \text { Dur } & \mathbf{R}=0,930^{*}
\end{array}
$$


On trouve bien des pourcentages d'explication très élevés (86\% au deuxième palier).

La dureté (méthode Janka) apparaît dans les équations de régression. Ainsi, cette caractéristique, rarement utilisée en France pour les recherches sur le bois, semble bien être celle qui présente le plus d'analogie avec les résultats obtenus au torsiomètre.

Le rôle moins important qu'elle joue dans l'explication du couple côté moelle peut surprendre ; ceci est dû vraisemblablement à la mise en jeu d'autres contraintes telles que le frottement exercé sur l'extérieur de la tarière après les épaulements terminaux des filets.

\section{5. - CONCLUSION}

\section{1. - Mode demploi}

L'utilisation d'un torsiomètre permet d'obtenir en forêt même, de façon simple et rapide, des renseignements très utiles sur la densité et les caractéristiques mécaniques du bois ; mais les conditions d'emploi doivent être bien définies, et les diverses expériences dont il est rendu compte ici ont mis en évidence l'influence, ou au contraire l'absence d'influence, d'un certain nombre de facteurs:

- La profondeur de mesure exerce un effet indiscutable sur le couple de torsion, en raison des frottements qui se produisent sur l'extérieur du corps de la tarière. Par ailleurs, on sait qu'un gradient de densité existe pour la plupart des essences résineuses de la moelle vers l'écorce ; il faut donc, sauf exception justifiée, prendre le couple de torsion toujours à la même distance à l'intérieur de l'arbre, et pour cela tenir compte de l'épaisseur de l'écorce qu'il convient donc de mesurer à l'aide d'une jauge appropriée. La modalité profonde, étudiée dans l'expérience * Sivrite 1 , $(3,75 \mathrm{~cm}$ à l'intérieur de l'arbre) semble, en règle générale, légèrement plus discriminante que la modalité superficielle.

- La lecture du torsiomètre ne doit pas se faire au démarrage de la tarière, mais un peu plus tard lorsque son aiguille s'est stabilisée, c'est-à-dire lorsqu'a cessé son déplacement relatif par rapport au cadran gradué ; on obtient ainsi une meilleure corrélation avec la densité et les écarts éventuels entre opérateurs ou entre tarières se trouvent minimisés.

- L'époque des mesures (sondages en sève ou hors sève) semble n'exercer aucun effet sur le couple de torsion.

- L'usure de la tarière ne se traduit pas, dans des conditions normales d'emploi, par une augmentation du couple de torsion, puisqu'on a même observé un effet contraire (non significatif pour le Grandis, mais significatif pour le Douglas) dans l'expérience s Sivrite 2 s, après une utilisation à vrai dire peu courante de l'instrument.

Un affûtage périodique des tarières garde par ailleurs tout son intérêt lorsque les échantillons recueillis doivent servir à d'autres études pour lesquelles un diamètre uniforme des carottes de sondage est un facteur important (retrait ou microdensitométrie). 
- L'homogénéité des résultats entre différents opérateurs ne peut étre considérée comme acquise puisque, si l'on n'a jamais observé de différences notables entre deux des expérimentateurs de * Sivrite 2 \% on a presque toujours obtenu des écarts significatifs entre eux et le troisième notateur ; il reste donc à harmoniser la vitesse de mise en cuvre du torsiomètre qui paraît être responsable de cet état de choses; il y a notamment intérêt à utiliser un appareil à long manche qui, à couple appliqué égal, nécessite une force moindre, ce qui facilite l'uniformisation des vitesses de rotation par des opérateurs de vigueur physique différente.

- On doit enfin mentionner l'existence de torsiomètres de sensibilités différentes ; il convient naturellement d'utiliser ceux qui sont le plus appropriés à la dureté des arbres sondés, par exemple, des torsiomètres d'une capacité de $300 \mathrm{~cm} . \mathrm{kg}$ pour la plupart des essences résineuses et de $600 \mathrm{~cm} . \mathrm{kg}$ pour les feuillus durs comme le Chêne ou le Hêtre : pour ces derniers, il y a en outre intérêt à employer des tarières spéciales à pas plus faible, donc nécessitant à dureté égale des couples de torsion moins élevés.

Au total, lorsque sont respectés les diverses conditions rappelées ci-dessus, la mesure des couples de torsion exercés pour enfoncer une tarière à une profondeur donnée permet d'obtenir aisément et de façon non destructive une première appréciation de la qualité du bois d'un grand nombre darbres in sín. Cette estimation peut d'ores et déjà être considérée comme valable en valeur relative, et le sera en valeur absolue lorsqu'on aura trouvé le moyen d'harmoniser automatiquement les vitesses de mise en mouvement des torsiomètres: il convient cependant de ne pas exagérer les difficultés dues à l'influence de l'opérateur qui peut éventuellement faire l'objet d'une correction appropriée, et la méthode garde en outre tout son intérêt lorsque les mesures sont faites par un seul expérimentateur ou par un groupe dont l'homogénéité a été préalablement vérifiée.

\section{2. - Applications pratiques}

Elles peuvent être nombreuses et variées.

Pour les chercheurs directement concernés par l'étude des aspects biologiques de la qualité du bois, le torsiomètre fournit une donnée supplémentaire qui s'ajoute à toutes celles que l'on peut déjà obtenir de façon non destructive, et qui apparaît de plus comme particulièrement discriminante pour analyser les effets de la station, de l'hérédité ou des diverses interventions humaines sur les caractéristiques technologiques du bois produit.

Lorsque l'on veut procéder à une étude plus complète nécessitant la réalisation d'essais sur éprouvettes normalisées, le torsiomètre permet de choisir, au sein d'un groupe d'arbres donné, un individu représentatif, c'est-à-dire présentant des caractéristiques voisines de la moyenne du groupe : cette possibilité ne doit pas être sousestimée car il n’existe actuellement aucun autre moyen de parvenir à ce résultat ; or la quantité de travail et le temps nécessaire pour l'obtention d'éprouvettes normalisées sont tels qu'il n'est pas possible d'analyser de la sorte un nombre suffisant pour prendre en compte la variabilité individuelle, et la plupart des expériences décrites dans la littérature reposent pour cette raison sur l'étude, très détaillée il est vrai, d'un seul 
arbre pour chaque traitement. Le coefficient de variation de nombreuses caractéristiques technologiques au sein d'une même parcelle unitaire pouvant atteindre $15 \%$, le risque est grand de tirer des résultats obtenus sur l'unique arbre d'essai des conclusions ne s'appliquant absolument pas à l'ensemble du groupe.

Un exemple récent de ce genre de danger peut être donné ici: une comparaison entre la qualité du bois de trois stations de Chêne zene de Tunisie a été demandée, d'une part à la Station de Recherches sur la Qualité des Bois du C.N.R.F. qui avait pour mission d'y procéder en utilisant une série de tests non destructifs, d'autre part à un Laboratoire spécialisé d'un pays d'Europe Orientale qui devait mener à bien un travail analogue au moyen d'essais sur éprouvettes normalisées issues d'un seul individu par station: les trois arbres abattus à cet effet avaient des densités s'écartant de la moyenne de la parcelle d'une quantité égale à - $74 \%$ de l'écart-type pour la première station, de $+56 \%$ pour la deuxième et de $-60 \%$ pour la troisième. Comment ne pas craindre que des conclusions erronées sur la valeur relative des trois stations ne soient tirées d'un tel échantillonnage?

D'une façon générale, l'utilisation de torsiomètres semblerait devoir faciliter et rendre plus efficace le travail de sélection, puisqu'il permettrait de choisir rapidement parmi des individus présentant d'autres caractères favorables: vigueur de croissance, rectitude du fût, finesse des branches, tardiveté du débourrement, etc..., ceux qui bénéficient en outre d'une densité satisfaisante; or la densité du bois apparaît, dans toutes les études de génétique quantitative, comme très héritable, chez les résineux tout au moins, et les gains de sélection à prévoir, lorsqu'on tient compte de ce critère, sont donc particulièrement élevés.

Il est même possible d'aller plus loin dans ce sens: J. MAdDERn Harris (1965). prenant en considération cette forte héritabilité, envisageait la possibilité d'améliorer la densité du bois par le seul moyen des pratiques sylvicoles traditionnelles: après la suppression, au cours des premières éclaircies, des tiges défectueuses ou à faible vigueur de croissance, il émettait l'idée particulièrement séduisante, tout au moins sur le plan théorique, d'une élimination, avant la coupe d'ensemencement, des arbres à faible densité, ce qui permettrait d'obtenir, dès la génération suivante, un peuplement amélioré sur le plan de la qualité du bois. Pour y parvenir, il appelait de ses voux la mise au point d'une méthode d'estimation automatique de la densité du bois sur une grande échelle, en forêt même ; le torsiomètre paraît bien répondre à cet objectif, et on peut envisager le jour où, dans certains pays particulièrement portés vers le progrès technique, il fera partie, au même titre que le compas ou le marteau, des outils de base du forestier,

Reçu pour publication en mars 1970.

\section{REMERCIEMENTS}

De nombreux et précicux concours nous ont été apportés au cours de cette étude :

M. Fondeur. Chef du Centre de Gestion de I'Office National des Forêts à St-Avold, nous a généreusement fourni les billons d'Epicéas utilisés pour les essais mécaniques de caractère destructif, dont l'échantillonnage a été fait par Fr. THIERCELIN, chercheur à la station, et dont le débit, particulièrement délicat, a été réalisé sous la direction de $\mathrm{R}$. Cochard. 
Pratiquement, tout le personnel de la station a participé à une phase ou à une autre du travail : expérimentation en forêt ou au laboratoire, calculs mathématiques simples ou présentation des résultats.

Enfin, nous naurions garde d'oublier laide très efficace de la Station de Biométrie du C.N.R.F.; nous sommes en particulier reconnaissants à C. MuLıer des études statistiques auxquelles il s'est livré, notamment pour lexpérience * Sivrite $2 \%$ où elles sortaient nettement du cadre des analyses de routine.

\section{SUMMARY}

FIRST APPRAISAL OF WOOD QUALITY \& IN SITU \& BY USING TORQUE MEASUREMENTS

In this paper are reported the results of a series of experiences concerning a new method of wood properties evaluation from standing trees, in which a torsiometer (fig. 2) is used to measure the torque required to drive an increment borer in the tree at a given depth.

The experience \& Sivrite 1 shows (table 1) that the best way (amongst the four which have been tested) for measuring the torsion torque is the so called * deep measurement after stabilization s, it means that the torsiometer is put on the head of the borer when it has penetrated the wood up to $3,75 \mathrm{~cm}$ after the end of the terminal shoulders of the threads and that the measurement is made when the torsiometer index shows no relative displacement on the dial. (table 2).

Elsewhere, the season (sapflow or resting period) has no effect on the torsion torque

In the experience Sivrite 2 s the effect of sharpening was studied by comparison between an increment borer very well sharpened (borer $\mathrm{n}^{\prime \prime}$ 1) and the same after a very intensive and continuous utilization by boring $3,000 \mathrm{~cm}$ within a dense hardwood (borer $\mathrm{n}$ " 2). The analysis of variance show no effect for the Grand Fir (table 4) and a significant effect for the Douglas Fir (table 3) but the torsion torque, contrary to common supposition, is lower for the strongly worn borer. This is probably due to an extreme burnishing of the borer during its quite unusual utilization and it may be concluded that for a normally used borer the effect of sharpening is negligible.

The influence of measurer has also been tested during this experience. When measurements are made after stabilization there is no effect in Douglas Fir (table 5) but significant effect in Grand Fir (table 6). This is apparently due to the different speeds used to turn the torsiometer, and can be corrected when necessary.

In the experience \& Sivrite 1 s and also in many others previously reported, the correlation between the torsion torque and the specific gravity was very narrow and in particular narrower than that observed between specific gravity and the different mechanical characteristics measured by standardized methods.

The experience \& St-Avold brings 2 explanations. On the one hand, the torsion torque is measured in wet conditions when standardized mechanical testings are made with samples in dry conditions. The tables 8 and 9 show that, using normalized samples in wet conditions, the correlations with specific gravity are much narrower. On the other hand, the step by step regression analysis shows that the specific gravity is correlated better with a combination of several mechanical characteristics (as is the torsion torque) than with only one of them.

Torsion torque which can be measured quickly and easily on a large number of trees is an additionnal data which is very discriminant to differenciate individuals when the study of biological aspects of wood quality is concerned.

When more detailed technological studies are to be undertaken, the torsiometer gives the alone mean to choose amongst a group of trees a sample tree having a density and a mechanical strength near to the mean value of the group.

At last the torsiometer seems to be a powerful tool for tree improvement, since it allows to select by non destructive testing from a plus stand having good others characteristics (quick growth, straightness, thin branches...) some trees also remarkable from the point of view of wood quality, particularly of specific gravity which is known to have a very high heritability. 


\section{ZUSAMMENFASSUNG}

\section{EINE ERSTABSCHĀTZUNG DER HOLZQUALITAT IM WALDE DURCH VERWENDUNG EINES} TORSIONSMESSERS

Es wird eine neue Methode zur Abschätzung der Holzqualität an lebenden Bäumen beschrieben. Sie beruht in der Verwendung eines Torsionsmessers zur Messung des Drehmoments welches zum Einbohren eines Pressler'schen Zuwachsbohrers bis zu einer bestimmten Tiefe notwendig ist.

Es wird über mehrere Versuche berichtet welche zur Erarbeitung einer geeigneten Messtechnik unternommen wurden. Der Versuch $*$ Sivrite I zeigte, dass von den vier untersuchten Methoden, die "Stabilisé profond (tab. 1) genannte Methode die besten Ergebnisse brachte. Dieser Versuch zeigte gleichfalls, dass der Zeitpunkt der Messung (Bäume in Saft oder ausser Saft) keinen Einfluss auf das Drehmoment ausübt.

Im Versuch \& Sivrite 2 , wurde der Einfluss des Schliffes der Zuwachsbohrer untersucht, wobei unter anderem ein feingeschliffener Zuwachsbohrer $\left(n^{-} 1\right)$ und derselbe $\left(n^{*} 2\right)$ nach einer intensiven Dauerbenutzung (3000 $\mathrm{cm}$ Bohrung in einem harten Holz) verglichen wurden. Es zeigten sich dabei signifikante Unterschiede zwischen den Drehmomenten in Douglasien jedoch nicht bei Abies grandis. Wider Erwarten waren jedoch die Drehmomente des Zuwachsbohrers $n^{*} 2$ (nach Dauerbeanspruchung) geringer, als jene des Zuwachsbohrers $n^{*} 1$ (vor Dauerbeanspruchung). Die anormale Beanspruchung des Bohrers n" 2 scheint eine gleichfalls aussergewöhnliche Polierung der Innenwand des Zuwachsbohrers hervorgerufen zu haben, wodurch die Reibung herabgesetzt wurde. Es folgt daraus, dass bei einem normal benutzten Zuwachsbohrer der Schliff keinen nennenswerten Einfluss ausübt.

Auch der Einfluss des Bohrspannehmers wurde im Versuch \& Sivrite 2 * untersucht. Erfolgen dic Messungen nach «Stabilisierung so zeigen sich signifikante Unterschiede zwischen den Bohrspannehmern bei der Küstentanne jedoch nicht bei der Douglasie. Die auftretenden Unterschiede sind offenbar auf die verschieden rasche Durchführung der Drehmomentmessung zurückzuführen und können notfalls korrigiert werden.

Im Versuch \& St-Avold * konnte geklärt werden, weshalb die Korrelationen zwischen der Dichte und dem Drehmoment enger sind als jene zwischen der Dichte und verschiedenen. an normalisierten Probestäben abgeschätzten mechanischen Merkmalen. Einerseits sind die Korrelationen mit der Dichte bei Prüfungen an waldgrünen Probestaben enger und anderseits ist der Eindringwiderstand des Zuwachshohrers sehr komplexer Natur (Kompression normal und parallel zu Holzfaserrichtung) und es zeigte sich tatsächlich, dass die Korrelationen zwischen der Dichte und einer Kombination von mechanischen Merkmalen enger sind als für jedes einzelne Merkmal.

Das Drehmoment ist daher ein neues Merkmal, welches leicht, rasch und nicht destruktiv an einer grossen Anzahl von Bäumen gemessen werden kann. Es können daher im Walde Bäume ausgewählt werden, die bezüglich Dichte und mechanischen Merkmalen den Bestandesmittelwerten entsprechen. Der Torsionsmesser kann durch die Auswahl in situ von Bäumen mit einer grösseren Holzdichte auch in der Forstgenetik Verwendung finden.

\section{REFERENCES BIBLIOGRAPHIQUES}

Madeler Harkis J, 1965. The heritability of wood density. I.U.F.R.O., Section 4I, Melbourne. Polge H., 1962. Recherches sur l'utilisation de prélèvements effectú́s à la tarière de Pressler pour l'étude des propriétés physiques et mécaniqués des bois. Rev. forest. fr., (10).

Polâ H. 1963. Contribution à létude de la qualité du bois des principales essances résineuses exotiques utilisées dans les reboisements français. Ann, Ec, nation. Eaux et Forểs et Stn. Rech. Expér., tome XX, fascicule 4. 